\title{
Características clínicas, epidemiológicas y manejo terapéutico de la meningitis pediátrica en dos instituciones de Medellín, Colombia
}

\author{
Clinical, epidemiological characteristics and therapeutic management of pediatric meningitis in two institutions of \\ Medellin, Colombia
}

\author{
Alejandra Uribe-Ocampo' orcid.org/0000-0003-3332-3562 \\ Sara Correa-Pérez ${ }^{1}$ orcid.org/0000-0003-1375-9983 \\ Libia María Rodríguez-Padilla2* orcid.org/0000-0001-7294-3735 \\ Juan Guillermo Barrientos-Gómez² orcid.org/0000-0001-5135-5168 \\ Juan Pablo Orozco-Forero² orcid.org/0000-0002-4166-4773
}

1 Semillero de Investigación, Facultad de Medicina. Universidad Pontificia Bolivariana. Medellín, Colombia.

2 Facultad de Medicina, Universidad Pontificia Bolivariana. Medellín, Colombia.

Uribe-Ocampo A, Correa-Pérez S, Rodríguez-Padilla LM, Barrientos-Gómez JG, Orozco-Forero JP. Características clínicas, epidemiológicas y manejo terapéutico de la meningitis pediátrica en dos instituciones de Medellín, Colombia. Univ. Salud. 2018;20(2):121-130. DOI: http://dx.doi.org/10.22267/rus.182002.116

\section{Resumen}

Introducción: La meningitis es una emergencia médica que requiere diagnóstico y tratamiento oportuno para evitar complicaciones. Objetivo: Determinar las características clínicas, epidemiológicas y el manejo terapéutico de meningitis en la primera infancia. Materiales y métodos: Estudio transversal que evaluó menores hasta los seis años de edad con meningitis bacteriana o aséptica en dos centros de Medellín, 2010 - 2013. Se recolectaron características clínicas, paraclínicas y terapéuticas. Las variables cualitativas se describieron mediante frecuencias absolutas y relativas y las cuantitativas con mediana y rango intercuartílico. Se exploró diferencias en las características de los pacientes según grupo de edad, mediante la prueba Chi-cuadrado o Fisher. Resultados: De 56 pacientes, $33(58,9 \%)$ eran del sexo masculino; $26(46,4 \%)$ presentaron meningitis bacteriana, $20(35,7 \%)$ aséptica y $10(17,9 \%)$ indeterminada; $36(64,3 \%)$ fueron mayores de dos meses, con manifestaciones clínicas inespecíficas (fiebre e irritabilidad). Se aislaron principalmente microorganismos Gram negativos en hemocultivos y Gram positivos en líquido cefalorraquídeo. El tratamiento antibiótico más frecuente fue cefalosporinas de tercerageneración en 13 (65\%) menores de 2 meses y 28 (77,8\%) mayores. Fallecieron dos pacientes y seis presentaron complicaciones. Conclusiones: la meningitis bacteriana fue la más frecuente en menores de un año. El tratamiento combinado es ideal para garantizar un adecuado cubrimiento y evitar complicaciones.

Palabras clave: Meningitis bacteriana; meningitis aséptica; cultivo de líquido cefalorraquídeo; niños. (Fuente: DeCS, Bireme).

\section{Abstract}

Introduction: Meningitis is a medical emergency that requires early diagnosis and treatment to avoid complications. Objective: To determine the clinical, epidemiological characteristics and therapeutic management of meningitis in early childhood. Materials and methods: A cross-sectional study, which evaluated children up to six years old with bacterial or aseptic meningitis from 2010 to 2013, was conducted in two centers in Medellin. Clinical, paraclinical and therapeutic characteristics were collected. The qualitative variables were described by absolute and relative frequencies, and the quantitative ones with median and interquartile range. Differences in patient characteristics according to age group were explored, using the Chi-square or Fisher test. Results: From the 56 patients studied, 33 
(58.9\%) were male; 26 (46.4\%) showed bacterial meningitis, 20 (35.7\%) aseptic one and $10(17.9 \%)$ indeterminate one; 36 (64.3\%) were older than two months, with non-specific clinical manifestations (fever and irritability). Gramnegative microorganisms were mainly isolated in blood cultures and Gram positive in cerebrospinal fluid. The most common antibiotic treatment was third-generation cephalosporins in 13 (65\%) children who were less than 2 months and $28(77.8 \%)$ in older ones. Two patients died and six children presented complications. Conclusions: Bacterial meningitis was the most frequent in children under one year old. Combined treatment is ideal to ensure adequate coverage and avoid complications.

Keywords: Meningitis, bacterial; meningitis, aseptic; cerebrospinal fluid culture; child. (Source: DeCS, Bireme).

\section{Introducción}

La meningitis es un síndrome neurológico de etiología bacteriana o aséptica. La meningitis bacteriana (MB) es causante de alta morbilidad y mortalidad, la última alcanza 12 a $50 \%$ en países subdesarrollados a pesar del tratamiento(1). Por el contrario, la meningitis aséptica (MA), frecuentemente de etiología viral, es autolimitada y se asocia con mejor pronóstico. La meningitis es más prevalente en países en vía de desarrollo y en edades extremas, presentándose frecuentemente en menores de cinco años. La sospecha clínica siempre debe estar presente, ya que el cuadro clínico se puede instaurar en menos de 24 horas hasta en un $25 \%$ de los niños y las formas de presentación fulminantes tienen mayor riesgo de mortalidad, por lo que los retrasos en la iniciación de la terapia antibiótica afectan negativamente el pronóstico(2,3).

En Colombia se desconocía la magnitud del problema, debido al bajo registro en el Sistema de Vigilancia en Salud Pública (SIVIGILA); sin embargo, este se ha fortalecido y para la semana epidemiológica 45 de 2015, se habían reportado 560 casos de MB, en 119 no se aisló microorganismo, pudiendo corresponder a los grupos de MA o meningitis indeterminada (MI). De 369 casos, $169(45,8 \%)$ corresponden a casos probables, $200(54,2 \%)$ a casos confirmados. MB probable y confirmadas fueron por: Neisseria meningitidis (N. meningitidis) 77 (20,9\%), Streptococcus pneumoniae (S. pneumoniae) 115 $(31,2 \%)$, Haemophilus influenzae (H. influenzae) $37(10,0 \%)$ y $140(37,9 \%)$ por otros agentes ${ }^{(4)}$.
De acuerdo a las características clínicas del paciente y los resultados del análisis citoquímico, tinción Gram y el cultivo del líquido cefalorraquídeo (LCR), se permite enfocar la sospecha en una MB o MA para determinar la antibioticoterapia ideal, que debe ser de amplio espectro y capaz de entrar rápidamente en el espacio subaracnoideo. El cultivo es el Gold Standar para realizar el diagnóstico de MB, sin embargo, en el $50 \%$ de los casos no se confirma crecimiento bacteriano(5), lo que enfoca el diagnóstico de MA de origen infeccioso (más frecuente los virus) o no infeccioso, sin embargo, en la mayoría de los casos (62\%), la etiología es incierta ${ }^{(6)}$.

El pronóstico a corto y largo plazo depende del diagnóstico precoz y oportuno, la identificación del agente causal y el tratamiento adecuado para evitar complicaciones, principalmente neurológicas, con un alto riesgo de morbilidad a largo plazo(7-9). El tratamiento recomendado por diversas guías internacionales incluye cefalosporina de tercera generación, ampicilina o penicilina $G$ que pueden usarse contra organismos susceptibles y a menudo se adiciona vancomicina al régimen empírico con las cefalosporinas(10,11). El uso concomitante de esteroides en neonatos reduce el riesgo de muerte principalmente en $\mathrm{MB}$ por $S$. pneumoniae(12), en niños mayores reduce la tasa de sordera y secuelas neurológicas a corto plazo, pero no se demostró disminución en la mortalidad en esta edad(13). El objetivo del presente estudio fue determinar las características clínicas, epidemiológicas y el manejo terapéutico de meningitis en la primera infancia en dos centros de referencia de Medellín, 2010 - 2013. 


\section{Materiales y métodos}

\section{Diseño y población del estudio}

Estudio transversal, que evaluó los pacientes pediátricos hasta los seis años de edad, con diagnóstico de meningitis, que fueron atendidos entre 2010 y 2013, en dos centros de tercer nivel de complejidad de la ciudad de Medellín. No se realizó ningún tipo de muestreo, se incluyó toda la población que cumpliera los criterios de elegibilidad del estudio.

Criterios de inclusión: Niños menores de 6 años de edad, con diagnóstico de meningitis bacteriana $o$ aséptica(14):

a) MB: cultivo en LCR positivo para microorganismo bacteriano, o sospecha si existe en LCR tinción de Gram positiva, recuento de glóbulos blancos en leucocitos $(\mathrm{WBC})>1000 / \mu \mathrm{L}$ con predominio de neutrófilos y concentración de glucosa en $\mathrm{LCR}<40 \mathrm{mg} / \mathrm{dL}$.

b) MA: Sospecha cuando se tiene WBC en LCR $<500 / \mu \mathrm{L}>50 \%$ células mononucleares, proteína en LCR < 80 a $100 \mathrm{mg} / \mathrm{dL}$, glucosa en LCR normal y tinción de Gram negativa.

Criterios de exclusión: Remisión del paciente a otra institución.

\section{Proceso de recolección de la información}

Se obtuvo a partir de la revisión de las historias clínicas y exámenes de laboratorio; se recolectaron variables sociodemográficas como sexo y edad; clínicas como antecedentes personales, antibiótico previo (3 meses antes del ingreso), signos y síntomas; parámetros hematológicos y de LCR; terapéuticas como tipo de tratamiento empírico y definitivo recibido y complicaciones a corto plazo, entre otros.

Para la clasificación del tipo de meningitis se consideró el diagnóstico de $\mathrm{MB}$ en quienes presentaron cultivo positivo y cambios en el citoquímico de LCR compatibles con infección bacteriana. MA como cambios en el citoquímico de LCR compatibles con inflamación meníngea no bacteriana sin aislamientos en los cultivos. MI o parcialmente tratada en quienes usaron antibiótico previo a la punción lumbar, con características en LCR de infección bacteriana, sin aislamiento en cultivos ${ }^{(5,15,16)}$.

Debido a las diferencias en la etiología y el tratamiento según la edad de los pacientes, estos se clasificaron en dos subgrupos: 1) menores e iguales a dos meses y 2) mayores de esta edad. Esta división facilitó el análisis de los episodios de meningitis en los pacientes puesto que una de las instituciones tiene una población de referencia que en su mayoría son neonatos y la otra atiende niños de todas las edades, pero principalmente mayores de 2 meses.

\section{Plan de análisis}

Las variables cualitativas se describieron con frecuencias absolutas y relativas $y$ las cuantitativas, como la edad, se expresó en mediana y rango intercuartílico (RIQ) dada la distribución heterogénea de los datos, según un resultado $\mathrm{p}<0,0001$ en la prueba de normalidad Kolgomorov-Smirnov. Para explorar si existían o no algunas diferencias en las características (antecedentes personales y manifestaciones clínicas) de los pacientes según el grupo de edad, se utilizó la prueba Chi cuadrado o Fisher cuando fue necesario (frecuencias esperadas inferiores a cinco). Los análisis estadísticos se llevaron a cabo con el programa SPSS versión 22.0.

\section{Consideraciones éticas}

Esta investigación fue clasificada como un estudio sin riesgo según Resolución 8430 de 1993 del Ministerio de Salud de Colombia, fue evaluada y aprobada por el Comité de Ética de las Instituciones participantes. Se analizó según los principios de beneficencia, no maleficencia, justicia y autonomía. La información fue obtenida directamente de historias clínicas respetando la confidencialidad de los datos personales de los pacientes(17). 


\section{Resultados}

Características sociodemográficas y clínicas

En las dos instituciones durante el período de estudio, se atendieron 74 pacientes pediátricos relacionados con el diagnóstico de meningitis, de estos 56 fueron seleccionados según los criterios de elegibilidad. De los cuales, 33 (58,9\%) pacientes eran del sexo masculino, la mediana de edad fue 4,1 meses (RIQ 0,6 - 11,7). La MB fue la más frecuente en $26(46,4 \%)$, seguida de la MA en $20(35,7 \%)$ y la indeterminada en $10(17,9 \%)$ niños.

Veinte $(35,7 \%)$ pacientes fueron menores e iguales a dos meses, y $36(64,3 \%)$ mayores de dos meses. La mayoría de pacientes menores, 12 (60\%), fueron atendidos en una de las instituciones, mientras que en la otra hubo mayor atención de pacientes mayores de dos meses, 31 (86,1\%). La frecuencia del tipo de meningitis según los grupos de edad, se muestran en la Figura 1.

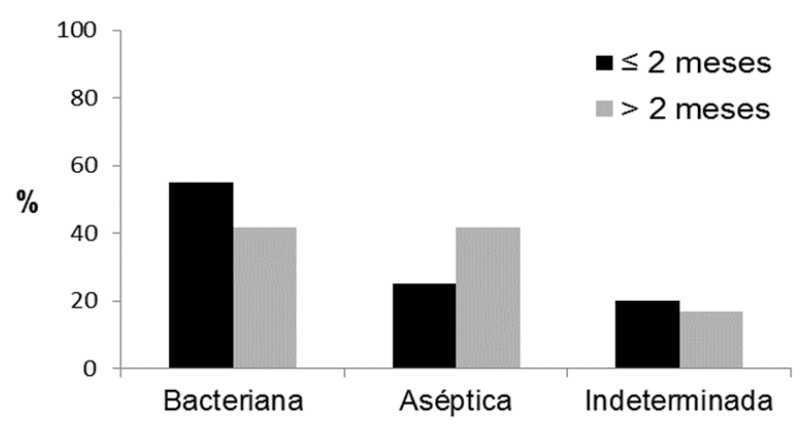

Figura 1. Tipos de meningitis por grupos de edad

La prematuridad y el bajo peso al nacer, fueron los antecedentes más frecuentes en el grupo de menores e iguales a dos meses comparados con los mayores de esta edad ( $\mathrm{p}<0,0001$ y $\mathrm{p}=0,007$, respectivamente). Por el contrario, en el grupo de mayores de dos meses, las infecciones respiratorias altas fueron las más frecuentes $(\mathrm{p}=0,01)$. En cuanto al uso de antibióticos previos, no hubo diferencias entre los grupos ( $\leq$ 2 meses: $35 \%$ vs. $>2$ meses: $41,2 \%, p=0,773)$. (Tabla 1). Ningún paciente tuvo inmunodeficiencias.

Tabla 1. Antecedentes personales según grupos de edad

\begin{tabular}{|c|c|c|c|c|}
\hline Antecedentes & $\begin{array}{c}\leq 2 \text { meses } \\
n / N(\%)\end{array}$ & $\begin{array}{c}>2 \text { meses } \\
n / N(\%)\end{array}$ & $\begin{array}{l}\text { Diferencia } \\
\text { proporciones } \\
\text { (IC95\%) }\end{array}$ & Valor p $\rrbracket^{\pi}$ \\
\hline \multicolumn{5}{|l|}{ Patológicos } \\
\hline Prematuridad & $11 / 20(55,0)$ & $4 / 36(11,1)$ & $44(16 ; 72)$ & $<0,0001$ \\
\hline Bajo peso al nacer & $7 / 20(35,0)$ & $2 / 36(5,6)$ & $29,4(3,4 ; 55)$ & $0,007 \ddagger$ \\
\hline Malformaciones Congénitas* & $3 / 20(15,0)$ & $5 / 36(13,9)$ & $1,1(-22,1 ; 24,3)$ & 1,0 \\
\hline Cardiopatía & $4 / 20(20,0)$ & $2 / 36(5,6)$ & $14,4(-8,5 ; 37,4)$ & $0,172 \ddagger$ \\
\hline Infección respiratoria alta reciente & $1 / 20(5,0)$ & $13 / 36(36,1)$ & $-31,1(-39,6 ; 17,4)$ & 0,010 \\
\hline \multicolumn{5}{|l|}{ Quirúrgicos } \\
\hline Cirugía del SNC & $1 / 20(5,0)$ & $9 / 36(25,0)$ & $-20(-41,0 ; 1,0)$ & $0,078^{\ddagger}$ \\
\hline Contacto Respiratorio & $3 / 19(15,8)$ & $6 / 27(22,2)$ & $-6,4(-33,6 ; 20,7)$ & 0,588 \\
\hline Uso de antibiótico previo & $7 / 20(35,0)$ & $14 / 36(41,2)$ & $-6,2(-36,8 ; 24,5)$ & 0,773 \\
\hline \multicolumn{5}{|l|}{ Tiempo de uso de antibiótico previo } \\
\hline Menos de una semana & $5 / 7(71,4)$ & $9 / 13+(69,2)$ & $2,2(-50,6 ; 68,2)$ & $1,0 \neq$ \\
\hline \multicolumn{5}{|c|}{$\begin{array}{l}\text { *Arnold Chiari, Mielomeningocele, Hidrocefalia, Otras. } \\
\text { † En un paciente no hubo disponibilidad del tiempo de uso de antibiótico previo. } \\
\text { †Prueba Fisher } \\
\text { ฯ Prueba Chi cuadrado }\end{array}$} \\
\hline
\end{tabular}

\section{Signos y síntomas}

La fiebre y el vómito se presentaron más frecuentemente en el grupo de los mayores comparados con el grupo de los menores de dos meses. A la vez, la irritabilidad fue el signo más común en los dos grupos, sin embargo, este signo fue más frecuente en los mayores de dos meses (Tabla 2). 
Asociado al episodio de meningitis, 30(53,5\%) niños tenían una infección concomitante, de los menores de dos meses 17(85\%) y de los mayores $13(36,1 \%)$ pacientes. Las infecciones más frecuentes fueron sepsis en $14(70 \%)$ en el primer grupo e infección urinaria en $5(13,9 \%)$ niños del segundo grupo.

Tabla 2. Manifestaciones clínicas según grupo de edad

\begin{tabular}{|c|c|c|c|c|}
\hline & $\begin{array}{c}\leq 2 \text { meses } \\
n / N(\%)\end{array}$ & $\begin{array}{c}>2 \text { meses } \\
n / N(\%)\end{array}$ & $\begin{array}{c}\text { Diferencia } \\
\text { proporciones } \\
\text { (IC95\%) }\end{array}$ & Valor $\mathbf{p}^{\dagger \dagger}$ \\
\hline \multicolumn{5}{|l|}{ Síntomas } \\
\hline Fiebre & $8 / 20(40)$ & $31 / 36(86,1)$ & $-46,1(-74,3 ;-18,0)$ & 0,001 \\
\hline Vómito & $2 / 20(10)$ & $24 / 36(66,7)$ & $-56,7(-80,8 ;-32,5)$ & 0,0001 \\
\hline Generales* & $7 / 20(35)$ & $7 / 36(19,4)$ & $15,6(-12,9 ; 33,4)$ & 0,98 \\
\hline Cefalea & 0 & $10 / 36(27,8)$ & $-27,8(-46,3 ;-9,3)$ & 0,010 \\
\hline Náuseas & 0 & $6 / 36(16,7)$ & $-16,7(-32,7 ;-0.6)$ & $0,078^{* *}$ \\
\hline Respiratorios superiores ${ }^{\dagger}$ & $2 / 20(10)$ & $2 / 36(5,6)$ & $4,4(-14,6 ; 23,5)$ & $0,611_{-}^{* *}$ \\
\hline \multicolumn{5}{|l|}{ Signos } \\
\hline Irritabilidad & $9 / 20(45)$ & $26 / 36(72,2)$ & $-27,2(-57,4 ; 2,9)$ & 0,084 \\
\hline Convulsión & $4 / 20(20)$ & $11 / 36(30,6)$ & $-10,6(-37,5 ; 16,4)$ & 0,393 \\
\hline Irritación meníngea ${ }^{\ddagger}$ & 0 & $13 / 36(36,1)$ & $-36,1(-55,7 ;-16,5)$ & 0.02 \\
\hline Fontanela abombada & $1 / 20(5)$ & $6 / 24(25)$ & $-20(-44,4 ; 4,4)$ & $0,159^{* *}$ \\
\hline Hipotonía & $3 / 20(15)$ & 0 & $15(-4,5 ; 34,5)$ & $0,06^{* *}$ \\
\hline Fotosensibilidad & 0 & $2(5,6)$ & $5,6(-16,9 ; 5,8)$ & $0,532^{* *}$ \\
\hline $\begin{array}{l}\text { * Malestar general, adinam } \\
\text { † Tos seca, rinorrea y otalg } \\
\text { * Signo de Kerning, Brudzir } \\
\text { † Característica de la fontar } \\
\text { ** Prueba Fisher } \\
\text { †† Prueba Chi cuadrado }\end{array}$ & $\begin{array}{l}\text { actividad y desh } \\
\text { aca. Aplica para l } \\
\text { res de } 18 \text { meses. }\end{array}$ & $\begin{array}{l}\text { atación } \\
\text { nenores de } 18 \mathrm{r}\end{array}$ & & \\
\hline
\end{tabular}

Tabla 3. Aislamiento de microorganismos en hemocultivo y cultivo de LCR

\begin{tabular}{|c|c|c|c|c|c|c|c|c|c|c|c|c|c|}
\hline \multirow{8}{*}{ 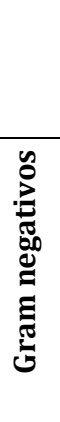 } & \multirow[b]{3}{*}{ E. coli } & \multicolumn{6}{|c|}{ Hemocultivos, n: 23} & \multicolumn{6}{|c|}{ Cultivos LCR, n: 22} \\
\hline & & \multicolumn{2}{|c|}{ n (\%) } & \multicolumn{2}{|c|}{$\begin{array}{c}\leq 2 \mathrm{~m} \\
\mathrm{n}: 14(\%)\end{array}$} & \multicolumn{2}{|c|}{$\begin{array}{c}>2 \mathrm{~m} \\
\mathrm{n}: 9(\%)\end{array}$} & \multicolumn{2}{|c|}{ n (\%) } & \multicolumn{2}{|c|}{$\begin{array}{c}\leq 2 \mathrm{~m} \\
\mathrm{n}: 8(\%)\end{array}$} & \multicolumn{2}{|c|}{$\begin{array}{c}>2 m \\
n: 14(\%)\end{array}$} \\
\hline & & 5 & $(21,7)$ & 2 & $(14,3)$ & 3 & $(33,3)$ & 3 & $(13,6)$ & 2 & $(25,0)$ & 1 & $(7,1)$ \\
\hline & H. influenzae & 3 & $(13,0)$ & 2 & $(14,3)$ & 1 & $(11,1)$ & 2 & $(9,1)$ & 1 & $(12,5)$ & 1 & $(7,1)$ \\
\hline & E. cloacae & 1 & $(4,3)$ & 1 & $(7,1)$ & & - & & - & & - & & - \\
\hline & E. faecalis & 1 & $(4,3)$ & 1 & $(7,1)$ & & - & 1 & $(4,5)$ & 1 & $(12,5)$ & & - \\
\hline & K. pneumoniae & & - & & - & & - & 1 & $(4,5)$ & & - & 1 & $(7,1)$ \\
\hline & Otros* & & - & & - & & - & 2 & $(9,1)$ & 1 & $(12,5)$ & 1 & $(7,1)$ \\
\hline \multirow{6}{*}{ 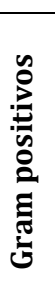 } & S. epidermidis & 2 & $(8,7)$ & 1 & $(7,1)$ & 1 & $(11,1)$ & 6 & $(27,3)$ & 1 & $(12,5)$ & 5 & $(35,7)$ \\
\hline & S. pneumoniae & 2 & $(8,7)$ & & - & 2 & $(22,2)$ & 3 & $(13,6)$ & & - & 3 & $(21,4)$ \\
\hline & S. agalactiae & 2 & $(8,7)$ & 2 & $(14,3)$ & & - & 1 & $(4,5)$ & 1 & $(12,5)$ & & - \\
\hline & S. hominis & 2 & $(8,7)$ & 2 & $(14,3)$ & & - & & - & & - & & - \\
\hline & S. aureus & 1 & $(4,3)$ & & - & 1 & $(11,1)$ & 2 & $(9,1)$ & & - & 2 & $(14,3)$ \\
\hline & Otros $^{\dagger}$ & 4 & $(17,4)$ & 3 & $(21,4)$ & 1 & $(11,1)$ & 1 & $(4,5)$ & 1 & $(12,5)$ & & - \\
\hline
\end{tabular}

Abreviaciones: 2m (2 meses)

* Otros Gram negativos: Enterobacter, Mycobacteria

† Otros Gram positivos: S. galloliticus, S. viridans, S. capitis, S. marcences 


\section{Hallazgos de laboratorio}

A 45 de los 56 pacientes, se les realizó hemocultivos, de éstos, 22 fueron positivos $(48,8 \%)$ y se aislaron 23 microorganismos; el más frecuente fue Escherichia coli (E. coli) identificado en 5/22 (22,7\%) hemocultivos, seguido por $H$. influenzae. En uno de los hemocultivos se aislaron dos microorganismos $S$. pneumoniae y Streptococcus epidermidis ( $S$. epidermidis). Se realizaron 56 cultivos de LCR, de los cuales $22(39,3 \%)$ fueron positivos, siendo $S$. epidermidis la bacteria más frecuentemente en $6 / 22(27,3 \%)$ cultivos, seguido por $E$. coli y $S$. pneumoniae (Tabla 3).

En los cultivos de LCR, las cepas de $S$. agalactiae, $H$. influenzae, E. faecalis y E. cloacae se aislaron sólo en menores de dos meses; la mayoría de $E$. coli y la totalidad de cepas de $S$. epidermidis, $S$. aureus y $S$. pneumonie se aislaron en mayores de dos meses. De los 22 cultivos de LCR que presentaron aislamiento microbiológico, 12 hemocultivos fueron positivos, en 11 casos se aisló el mismo microorganismo tanto en cultivo de LCR como en hemocultivo y solo en un caso hubo diferencias en el aislamiento, encontrando $S$. viridans en el hemocultivo y $S$. epidermidis en cultivo de LCR.

Según el antibiograma, de los 23 hemocultivos positivos, las cepas bacterianas que mostraron mayor resistencia fueron $E$. coli, resistentes a ampicilina/sulbactam, gentamicina, y trimetoprim/sulfametoxazol (TMP/SMX). En los hemocultivos, los microorganismos que no presentaron resistencia a ningún antibiótico fueron: $H$. influenzae, S. pneumoniae, S. aureus, $S$. galloliticus, E. faecalis y S. capitis. En los 22 cultivos de LCR, $S$. epidermidis fue el microorganismo que presentó mayor resistencia, siendo resistente a eritromicina, oxacilina, clindamicina, TMP/SMX, rifampicina y a tetraciclinas (Tabla 3). No se identificaron patrones de resistencia como BLEES, AmpC o resistencia a la vancomicina, pero en $S$. epidermidis se observó resistencia a oxacilina.

Tabla 4. Tratamiento empírico y definitivo

\begin{tabular}{|c|c|c|}
\hline & $\begin{array}{c}\leq 2 \text { meses } \\
\text { n }(\%)\end{array}$ & $\begin{array}{c}>2 \text { meses } \\
\text { n (\%) }\end{array}$ \\
\hline Tratamiento Empírico & $20(100)$ & $33(100)$ \\
\hline Glucopéptidos & $4(20)$ & $29(80,6)$ \\
\hline Cefalosporinas $3^{\mathrm{a}}$ y $4^{\mathrm{a}}$ generación & $13(65)$ & $28(77,8)$ \\
\hline Penicilina & $11(55)$ & $2(5,6)$ \\
\hline Aminoglicósidos & $9(45)$ & $2(5,6)$ \\
\hline Penicilina/ inhibidor de betalactamasa & $3(15)$ & $2(5,6)$ \\
\hline Carbapenem & $3(15)$ & $2(5,6)$ \\
\hline Antiviral & $0(0)$ & $5(13,9)$ \\
\hline \multicolumn{3}{|l|}{ De tratamiento empírico a definitivo } \\
\hline Sin ajuste en el tratamiento & $2(10)$ & $6(18,2)$ \\
\hline Ajuste en el tratamiento & $18(90)$ & $27(81,8)$ \\
\hline - Cambio todo el tratamiento & $12(60)$ & $20(60,6)$ \\
\hline - Remoción & $6(30)$ & $5(45,5)$ \\
\hline - Reajuste en dosis & $0(0)$ & $2(6,1)$ \\
\hline Tratamiento definitivo & $18(90)$ & $30(83,3)$ \\
\hline Cefalosporinas $3^{\text {a }}$ y $4^{\text {a }}$ generación & $9(45)$ & $19(52,8)$ \\
\hline Glucopéptidos & $6(30)$ & $19(52,8)$ \\
\hline Penicilina & $6(30)$ & $3(8,3)$ \\
\hline Carbapenem & $4(20)$ & $5(13,9)$ \\
\hline Antimicóticos & $4(20)$ & $1(2,8)$ \\
\hline Aminoglicósidos & $4(20)$ & $1(2,8)$ \\
\hline Total días de tratamiento & $15,5(12,2-22)^{*}$ & $14(10-22)^{*}$ \\
\hline
\end{tabular}




\section{Tratamiento}

De los 56 pacientes, 53 (94,6\%) recibieron tratamiento con algún antimicrobiano o antiviral. Los antibióticos más usados fueron cefalosporinas de tercera y cuarta generación, principalmente ceftriaxona y cefepime, siendo utilizadas de manera empírica en 13 (65\%) menores de 2 meses y $28(77,8 \%)$ mayores. En ambos grupos, 18 (90\%) y $27(81,8 \%)$ niños respectivamente, requirieron ajuste de terapia (Tabla 4). Como complemento al tratamiento, 25 $(44,6 \%)$ pacientes recibieron dexametasona; 5 (25\%) en menores de dos meses y $20(55,6 \%)$ en mayores.

Dos pacientes murieron durante el tratamiento, los dos casos fueron en menores de 2 meses, correspondientes a un 3,6\% de la población total. Estos dos pacientes tuvieron una rápida evolución del cuadro clínico; en uno de ellos no se le identificó ningún microorganismo y al otro paciente se le aisló E. cloacae.

Las complicaciones a corto plazo se presentaron en $6(10,7 \%)$ pacientes; de estos, cinco casos fueron en el grupo de mayores de 2 meses (empiema en dos pacientes; choque séptico, infarto occipital con transformación hemorrágica y retraso del neurodesarrollo, cada una en un paciente). Sólo un paciente menor de 2 meses presentó coagulación intravascular diseminada.

\section{Discusión}

La meningitis causa alta morbilidad y mortalidad en países en vía de desarrollo, situándose en una de las 10 principales causas de muerte por enfermedades infecciosas en el mundo, después de las infecciones respiratorias y la enfermedad diarreica, como lo menciona el estudio de Souza et al.(18); por lo tanto, requiere un diagnóstico y tratamiento oportuno, para evitar complicaciones. A nivel mundial, se reporta una mayor frecuencia de la meningitis en menores de cinco años, por lo que, se decidió caracterizar dicho rango de edad en este estudio, agrupándose en menores y mayores a dos meses; para una mejor diferenciación de la clínica, microbiología y tratamiento. La mayoría de pacientes son menores a un año y de sexo masculino, similar a estudios como Silvestre et al., y Flórez et al.(19,20).

La MB fue el tipo más frecuente, acorde con lo sugerido por Antoniuk et al.(16), principalmente en menores de un año en países en vía de desarrollo, por su mayor susceptibilidad a infecciones bacterianas graves(21). Sin embargo, en la población preescolar se presenta más la MA, principalmente por virus, ya que la frecuencia de los principales microorganismos causantes de meningitis en este rango de edad han disminuido por la cobertura en la vacunación(16,22).

Aproximadamente la mitad de los pacientes requirieron uso de antibióticos previo al episodio de meningitis, lo cual puede aumentar la resistencia a los antibióticos y la negativización de los cultivos. Adicionalmente, las dificultades en las técnicas de laboratorio, también limita un adecuado diagnóstico, identificación del microorganismo y la elección de la terapia $(2,22,23)$.

Las manifestaciones clínicas más frecuentes son fiebre, irritabilidad y vómito; otras según Lewis et al., son hipotonía e hipoactividad(24). Estas características clínicas se consideran inespecíficas del cuadro de meningitis, especialmente en menores de 18 meses, que representaban la mayoría de la población de estudio. En contraste al estudio de Theodoridou et al.(25), en pacientes con mediana de dos años de edad, donde la mayoría presentaron fiebre y signos meníngeos, por lo que presentaban la clínica típica de este síndrome. Es claro que hay que tener alto índice de sospecha para diagnosticar la meningitis, debido a que los signos y síntomas dependen de la edad, la duración de la enfermedad y la respuesta a la infección(26).

Los microorganismos aislados en el LCR con mayor frecuencia fueron $E$. coli y $S$. epidermidis, siendo comunes en la población menor a un año, acorde con el estudio de Kim et al.(26); aunque $S$. epidermidis comúnmente no es un microorganismo causante de meningitis, se presentó en los pacientes que tenían algún factor de riesgo como cirugía en el sistema nervioso o 
estaban hospitalizados previo al episodio, hecho que explica este hallazgo. En varios cultivos de LCR en menores de dos meses, se aisló $H$. influenzae coincidiendo con la edad en que aún los pacientes no han recibido vacuna para éste. En contraste con la meningitis en la edad preescolar, los más frecuentes son $S$. pneumoniae y $N$. meningitidis y en menor medida el $H$. influenzae, por el impacto del programa ampliado de inmunización(27,28). Adicional al cultivo de LCR, el hemocultivo se realizó en la mayoría de la población, lo cual permitió identificar con mayor probabilidad al microorganismo en su fase de diseminación hematógena previa a la invasión meníngea; como se reporta en otros estudios como el de Ciapponi et al., existe un alto porcentaje de bacteriemia en niños con $\mathrm{MB}$ especialmente menores de 5 años $(29,30)$.

Los antibióticos más usados fueron cefalosporinas de tercera y cuarta generación, seguidos por el glucopéptido vancomicina, tanto de manera empírica como manejo definitivo acorde con las recomendaciones de las guías de IDSA (Sociedad de Enfermedades Infecciosas de América)(10) y en estudios como Ouchenir et al.(31), estos autores consideran usar carbapenems o ampicilina en la MB cuando el patógeno es desconocido y la tinción de Gram es negativa o muestra un organismo Gram negativo. Saraiva et al.(32), de acuerdo a la etiología en la MA sugieren uso de terapia antiviral, como se describe en el 13,9\% de la población de éste estudio. Se recomienda iniciar con un tratamiento combinado para cubrir los principales microorganismos dependiendo de la edad, la etiología local, y la resistencia a antibióticos(25,33).

En nuestro estudio, la dexametasona fue utilizada en menos de la mitad de los pacientes; según estudio de Browder et al., los esteroides son menos eficaces en países en desarrollo, donde hay gran prevalencia de malnutrición que genera mayores efectos adversos, la falta de administración temprana de medicamentos, y por el diagnóstico tardío(33). Cabe resaltar que los mayores de dos meses fueron quienes recibieron en mayor proporción la terapia con esteroides, pues en los pacientes menores de 2 meses no existe evidencia clara de disminución de secuelas neurológicas(26).

Las complicaciones agudas se presentaron en el $10,7 \%$ de pacientes, en contraste con el estudio de Flórez et al, que se presentaron en el $43 \%$, las cuales fueron empiema cerebral, choque séptico e infarto cerebral; además de estas, se reportan en la literatura otras complicaciones neurológicas como convulsiones, hidrocefalia, colecciones cerebrales, déficit focal, pudiendo ocurrir entre 3,3\% y $30 \%$ en pacientes con $\mathrm{MB}$, las cuales se presentan principalmente en pacientes de menor edad(25,34,35).

La letalidad de meningitis, en el estudio fue 3,6\%, similar a la de Lewis et al. $(3,8 \%)$ en Perú y en contraste con los estudios de Ouchenir en Canadá $14 \%{ }^{(31)}$ y Dueguer et al. en Guatemala cuya tasa fue de $27,6 \%{ }^{(36)}$; la baja mortalidad presentada en el presente estudio posiblemente se deba al alto nivel de complejidad de las instituciones, al oportuno diagnóstico y al inicio temprano del tratamiento.

En cuanto a las limitaciones inherentes a la investigación retrospectiva, las fuentes y los procedimientos de recolección de la información, hubo datos faltantes, como el registro de vacunación, esto tiene gran importancia al ser enfermedades infecciosas potencialmente prevenibles.

Se realizó el diagnóstico etiológico de la MA en dos casos, uno de ellos fue citomegalovirus y el otro caso fue enterovirus; en estudios como el de Saraiva et al., se observa un mayor uso de las pruebas moleculares(32), que permitió identificar el agente etiológico de la MA en el 29,7\% de sus casos, algo similar a los estudios de Dueguer et $a l .(36)$ y Conca et al.(37).

A pesar de que en ambas instituciones existía disponibilidad para realizar estudios moleculares, se observa que estas pruebas no se realizaron en la mayoría de los pacientes con sospecha de etiología viral; en vez de esto, a quienes no tuvieron aislamiento bacteriano se les realizó un manejo sindromático, dada la 
eficacia de las pruebas moleculares, es necesario aprovechar esta tecnología puesto que beneficia al paciente facilitando el diagnostico etiológico, repercutiendo en la clínica y en decisiones terapéuticas; así mismo, disminuye el uso de antibióticos y días de hospitalización y genera mayores beneficios en costo-efectividad(37).

En las instituciones hubo variabilidad en el grupo de antibióticos preseleccionados para realizar el antibiograma; para permitir mayor direccionamiento en las acciones y elección de terapia más oportuna, se deberían actualizar los protocolos para el estudio del antibiograma en cada una de las instituciones. Otra limitante, es el tamaño reducido de la muestra, lo cual afecta la precisión de los resultados, que se evidencia por intervalos de confianza amplios en las diferencias encontradas entre los grupos de población evaluados.

Este estudió permite conocer acerca de la epidemiología local teniendo como referencia dos centros de alto nivel de complejidad; las características clínicas y los microorganismos más frecuentes en éstas instituciones, así como el perfil de resistencia antimicrobiana, que aunque pueden tener diferencias con la etiología en otros niveles de atención, permiten aproximarse al manejo de la meningitis en pacientes pediátricos y sugerir alternativas de acuerdo a la disponibilidad de medicamentos en los diferentes centros de atención. Futuras investigaciones pueden enfocarse en el pronóstico a largo plazo de acuerdo con el tratamiento y diagnóstico realizado en las instituciones de salud, relacionando así mismo la vacunación con el cambio en la epidemiología local.

\section{Conclusión}

Las manifestaciones clínicas en menores de dos años son inespecíficas del cuadro de meningitis, de ahí radica la importancia de la sospecha clínica en esta población. En la mayoría de casos no se identifica agente etiológico, por lo tanto, para incrementar la posibilidad del diagnóstico se debe tener participación tanto de las características clínicas, como de estudios hematológicos, cultivos y pruebas moleculares para determinar oportunamente la etiología para MB o MA. El tratamiento de primera línea para la MB tanto empírico como definitivo es con cefalosporinas de tercera y cuarta generación acompañado la mayoría de veces con vancomicina para mejorar la penetración al SNC. Para la MA el tratamiento es el manejo sindromático y en casos específicos terapia antiviral.

Es importante la sospecha clínica de meningitis para diagnosticarla oportunamente y guiar su manejo. Conocer la etiología, resistencia antimicrobiana y características demográficas de la población local lleva a la realización de un tratamiento óptimo, pero se requieren más estudios con la participación de más instituciones y ciudades, para lograr caracterizar epidemiológicamente mejor esta enfermedad.

\section{Conflicto de interés}

Los autores declaran no tener ningún tipo de conflicto de interés.

\section{Referencias}

1. Nazia Khan, Abida Malik, Meher Rizvi, et al. Epidemiology and drug resistance profile of acute bacterial meningitis in children in Northern India: a university hospital perspective. Asian Pac J Trop Dis. 2014;4:S818-S823.

2. Bustos JE. Estudio epidemiológico y aplicación del bms (bacterial meningitis score) en los pacientes con diagnóstico de meningitis en el hospital de la misericordia en el año 2010. Bogotá: Universidad Nacional de Colombia; 2011. Disponible en: http://www.bdigital.unal.edu.co/6488/1/5598462.201 1.pdf

3. Hoz F, Duran MEM, García OEP, et al. Protocolo de Vigilancia en Salud Pública. Meningitis Bacteriana. Bogotá: Instituto Nacional de Salud; 2014.

4. SIVIGILA. Boletín epidemiológico, Semana epidemiológica número 45. Bogotá: Instituto Nacional de Salud; 2015.

5. Salgado MM, Gonçalves MG, Fukasawa LO, et al. Evolution of bacterial meningitis diagnosis in São Paulo State-Brazil and future challenges. Arq Neuropsiquiatr. 2013;71:672-676.

6. Shukla B, Aguilera EA, Salazar L, et al. Aseptic meningitis in adults and children: Diagnostic and management challenges. J Clin Virol Off Publ Pan Am Soc Clin Virol. 2017;94:110-114.

7. Sharon E. Mace. Acute bacterial meningitis. Emerg Med Clin North Am. 2008;26:281-317, viii. 
8. Køster-Rasmussen R, Korshin A, Meyer CN. Antibiotic treatment delay and outcome in acute bacterial meningitis. J Infect. 2008;57:449-454.

9. Martinez E, Mintegi S, Vilar B, et al. Prevalence and predictors of bacterial meningitis in young infants with fever without a source. Pediatr Infect Dis J. 2015;34:494-498.

10. Tunkel AR, Hartman BJ, Kaplan SL, et al. Practice Guidelines for the Management of Bacterial Meningitis. Clin Infect Dis. 2004;39:1267-1284.

11. Amy M. Pick, Desirae C. Sweet, Kimberley J. Begley. A Review of Pediatric Bacterial Meningitis. US Pharm. 2016;41:41-45.

12. Ogunlesi TA, Odigwe CC, Oladapo OT. Adjuvant corticosteroids for reducing death in neonatal bacterial meningitis. In: Cochrane Database of Systematic Reviews. John Wiley \& Sons, Ltd. Epub ahead of print 11 September 2011. DOI: 10.1002/14651858.CD010435.pub2.

13. Brouwer MC, McIntyre $\mathrm{P}$, Prasad $\mathrm{K}$, et al. Corticosteroids for acute bacterial meningitis. Cochrane Database Syst Rev. 2015; CD004405.

14. Di Pentima C. Viral meningitis: Management, prognosis, and prevention in children. Nueva Jersey: UpToDate; $2017 . \quad$ Disponible en: https://www.uptodate.com/contents/viral-meningitismanagement-prognosis-and-prevention-in-children (accessed 13 March 2018).

15. Baquero F, Vecino R, Castillo F. Meningitis bacteriana. Protoc Diagnóstico-Ter AEP Infectol Pediátrica. 2011;3:47-57.

16. Antoniuk S, França M, Tahan T, et al. Estudio de 312 niños con meningitis atendidos en un Hospital Universitario en el Sur de Brasil. Actual En Neurol Infant II. 2009;69:127-132.

17. Ministerio de Salud. Resolución Número 8430 de 1993, Por la cual se establecen las normas científicas, técnicas y administrativas para la investigación en salud. Bogotá: MinSalud; 1993.

18. Souza SF de, Costa M da CN, Paim JS, et al. Bacterial meningitis and living conditions. Rev Soc Bras Med Trop. 2012;45:323-328.

19. Silvestre JJ, Izquierdo A, Uscátegui A, et al. Características clínicas y paraclínicas de la meningitis bacteriana en niños. Acta Neurol Colomb. 2007;23:6-14.

20. Flórez O, Andrea J, Navas G, et al. Características clínicas y paraclínicas de niños con meningitis bacteriana aguda en el Hospital Universitario San Vicente Fundación en Medellín, Colombia. 2011 - 2015: estudio descriptivo - retrospectivo. Acta Neurológica Colomb. 2017;33:84-93.

21. Castellón SM, Ghetti EDP, Ortiz YM, et al. Validez del Score de Boyer para el diagnóstico diferencial de meningitis bacteriana y no bacteriana en niños del Hospital Manuel Ascencio Villarroel. Cochabamba, Bolivia. Gac Médica Boliv. 2016;38:1-6.

22. Rosario H. Zambrano-Silva, Carlos A. Vizueta-Chávez, Byron 0. López-Silva, et al. Incidencia de meningitis aguda en pacientes pediátricos del hospital "Francisco de Ycaza de Bustamante" durante el año 2010. Dominio Las Cienc. 2017;3:104-122.
23. Patiño RO, Bruges J, Lux AM, et al. Meningitis bacteriana aguda en niños: estudio clínico y bacteriológico en el Hospital Infantil de Medellín. Iatreia. 1988;1:69-76.

24. Lewis G, Schweig M, Guillén-Pinto $D$, et al. Neonatal meningitis in a general hospital in Lima, Peru, 2008 to 2015. Rev Peru Med Exp Salud Pública. 2017;34:233238.

25. Kalliopi Theodoridou, Vasilopoulou VA, Katsiaflaka A, et al. Association of treatment for bacterial meningitis with the development of sequelae. Int J Infect Dis. 2013;17(9): e707-713.

26. Kim KS. Acute bacterial meningitis in infants and children. Lancet Infect Dis. 2010;10:32-42.

27. Paradowska-Stankiewicz I, Piotrowska A. Meningitis and encephalitis in Poland in 2013. Przegl Epidemiol. 2015;69:229-234.

28. Castelblanco RL, Lee M, Hasbun R. Epidemiology of bacterial meningitis in the USA from 1997 to 2010: a population-based observational study. Lancet Infect Dis. 2014;14:813-819.

29. Pinzón AMÁ, Rodríguez-Martínez M, Charria-Ortiz G. Actualización en el tratamiento de la meningitis neonatal bacteriana y Reporte de un caso. Rev Med. 2010;18:100-114.

30. Ciapponi A, Elorriaga N, Rojas JI, et al. Epidemiology of Pediatric Pneumococcal Meningitis and Bacteremia in Latin America and the Caribbean: A Systematic Review and Meta-analysis. Pediatr Infect Dis J. 2014;33:971.

31. Ouchenir L, Renaud C, Khan S, et al. The Epidemiology, Management, and Outcomes of Bacterial Meningitis in Infants. Pediatrics. 2017;140:e20170476.

32. Saraiva MGG, Santos ECS, Saraceni V, et al. Epidemiology of infectious meningitis in the State of Amazonas, Brazil. Rev Soc Bras Med Trop. 2015;48:7986.

33. Kameshwar Prasad, Neha Karlupia, Amit Kumar. Treatment of bacterial meningitis: an overview of Cochrane systematic reviews. Respir Med. 2009;103:945-950.

34. Sadie Namani, Zvonko Milenković, Bulëza Koci. A prospective study of risk factors for neurological complications in childhood bacterial meningitis. J Pediatr (Rio J). 2013;89:256-262.

35. Davenport MC, Valle D, Paz M de la, et al. Meningitis bacteriana: factores de riesgo para el desarrollo de complicaciones agudas. Arch Argent Pediatría. 2007;105:405-410.

36. Dueger EL, Asturias EJ, Halsey NA. Culture- and antigennegative meningitis in Guatemalan children. Meningitis Negativa Pruebas Antigénicas Cultivo En Niños. Guatem. 2008;24:248-255.

37. Conca N, Santolaya ME, Farfan MJ, et al. Diagnóstico etiológico en meningitis y encefalitis por técnicas de biología molecular. Rev Chil Pediatría. 2016;87:24-30. 